\title{
Quasi-Stationarity of Electric Power Grid Dynamics Based on a Spatially Embedded Kuramoto Model
}

\author{
Herbert Mangesius, Sandra Hirche, and Dragan Obradovic
}

\begin{abstract}
A novel and simple network model that is capable to reproduce quasi-stationary behavior and propagation phenomena in electric power grid dynamics is introduced. A new Kuramoto approximation to distributed generator dynamics is obtained from combining a continuous spatial interaction function with a discrete lattice model representing generator positions and network structure over a continuous spatial domain. At hand of model properties and a numerical study quasi-stationarity of electric power grid dynamics results, being related to non-vanishing fluctuation and oscillations in stationary state.
\end{abstract}

\section{INTRODUCTION}

Developing innovative distributed controls is considered as major challenge to guarantee operational stability of the future bulk power supply. Solving this control problem rests on acquiring an understanding of global dynamic mechanisms in order to be able to define appropriate dynamic coordination of spatially separate local controls. Comprehension of global dynamics essentially resorts to finding conceptual models that explain diverse observed spatio-temporal phenomena, as for instance presented in [1]: so-called "global European modes" extend over the spatial domain of continental Europe, representing non-homogeneous vector fields that induce rotatory power flow over the whole spatial domain; their shape, capacity, and frequency characteristics are assumed to strongly depend on the large geographical scale, and to affect operational stability.

Electric power grid dynamics are usually studied as system of differential algebraic equations

$$
\begin{aligned}
\dot{\boldsymbol{x}} & =\boldsymbol{f}(\boldsymbol{x}, \boldsymbol{y}), \\
0 & =g(\boldsymbol{x}, \boldsymbol{y}),
\end{aligned}
$$

where the state dynamics 1a induce a flow on a compact manifold defined by $g$. Algebraic variables are assumed to be kept at a stationary value $\boldsymbol{y}^{s}$, due to local control of reference frequency, or voltage. Physically, 1a and 1b interact and evolve continuously, producing dynamics over the whole geographical scale of the network. One way to model such a behavior is by partial differential equations (PDEs). Simple PDE models have been proposed to acquire a deeper understanding of electric power system behavior,

H. Mangesius is with the Institute for Advanced Study (IAS), Technische Universität München (TUM), Lichtenbergstrasse 2a, D-85748 Garching. S. Hirche is with IAS and the Insitute of Automatic Control Engineering, TUM, Arcisstrasse 21, D-80290 Munich. D. Obradovic is with IAS, and Siemens AG Corporate Technology, Otto-Hahn-Ring 6, D-81739 Munich. mangesiusetum.de, hirche@tum.de, dragan.obradovicesiemens.com. With the support of the TUM-IAS, funded by the German Excellence Initiative. see [2], [3]. These models are capable to reproduce observed phenomena such as electromechanical wave propagation at finite speed (order $10^{2} \mathrm{~km} / \mathrm{s}$ ), but lack detailed insight on their origin and the role of local dynamics together with an interconnection structure. Simple network models, on the other hand, use local descriptions of dynamics and the interaction is described by a weighted graph that represents the electric interconnection structure [4], [5]. These interaction types rely on assumptions such as constant impedance loads, and constant power flow or voltages [5]-[7]. A general concern about dynamic consistency of such electric power grid models is raised in [7], due to interaction of the transmission system phasor dynamics with load phasor dynamics bearing the possibility to cause instability.

In this paper we propose a spatially embedded network model of Kuramoto oscillators that mimics the electric power grid dynamics close to an equilibrium solution. Local oscillators are ordered in a regular lattice. The interaction of local dynamics ensues in a mean field approach through the aggregate system. The effect of varying network variables is examined by means of a numerical simulation. The simulations indicate that dynamics converge rather to an oscillatory motion than to a static equilibrium characterized by (1).

The remainder of this paper is organized as follows: The classical equations of electric power grid dynamics are presented in II and enhanced by a spatial context. A Kuramoto approximation as special instance of diffusively coupled oscillators is given. In III the spatially embedded Kuramoto model is introduced and spatio-temporal solution properties are examined. In IV transient and stationary dynamics are studied, and generic trade-offs between reactivity and robustness are discussed on the basis of numerical simulations.

Notation: A graph $\mathcal{G}(\mathcal{V}, \mathcal{E})$ comprises of vertices indexed as $i, k \in \mathcal{V}$ (also called buses), and edges $i k \in \mathcal{E}$ (also called branches), and $k \mid i k \in \mathcal{E}$ refers to indices $k$ that are adjacent to $i$. A dynamic variable indexed as $(\cdot)_{i k}$ denotes difference $(\cdot)_{i}-(\cdot)_{k}$, a function of dynamic variables, and static parameters indexed as such denote property across the branch $i k$. The sets $\mathbb{C}, \mathbb{R}, \mathbb{Z}$, and $\mathbb{N}$ refer to the set of complex, real, integer, and natural numbers. Complex valued variables are indicated by capital letter with an upper bar, $j:=\sqrt{-1}$, and $(\cdot)^{*}$ denotes the complex conjugate. By that $\bar{Z} \in \mathbb{C}, \bar{Z}=a+j b, a, b \in \mathbb{R}$, in phasor notation is $Z \angle \vartheta:=$ $Z e^{j \vartheta}, Z:=|\bar{Z}|=\sqrt{a^{2}+b^{2}}, \vartheta:=\operatorname{atan} 2(b, a)$. Capital letter without bar denotes magnitude. Variables at certain (initial) time $t_{0}$, are associated to a stationary solution of (1), and they are marked as $(\cdot)^{0}$. Bold symbols denote vector valued 
quantities. The function $d(\cdot, \cdot)$ denotes distance, and $\|\cdot\|$ represents the Euclidean norm.

\section{REPRESENTATION AND DYNAMICS OF ELECTRIC POWER SYSTEMS}

The standard model approximation of the physical networked dynamics in electric power systems is presented and enhanced by a spatial extend. A Kuramoto approximation to the classical electric power system description is given in the context of diffusively coupled oscillator dynamics.

\section{A. Spatially Enhanced Classical Model Equations}

The basis for electric network studies is power balance, that is achieved by solving the complex power flow (or load flow) problem which has the form (1b). The complex power $\bar{S}_{i}=P_{i}+j Q_{i}=\bar{V}_{i} \bar{I}_{i}^{*}$, where $P$ is active and $Q$ reactive power, and $\bar{V}$ and $\bar{I}$ are voltage and current. Representing the electric network as graph $\mathcal{G}$, the power flow problem is to balance power injections at each network bus with power flow across branches in dependence of the electric network parameter admittance $\bar{Y}=Y e^{j \gamma}=G+j B$, where $G$ denotes conductance, $B$ susceptance, and voltages $V_{i} \angle \Theta_{e, i}$ at buses

$$
\begin{aligned}
P_{e, i}^{0} & =\sum_{k \mid i k \in \mathcal{E}} V_{i}^{0} V_{k}^{0}\left[G_{i k} \cos \Theta_{e, i k}^{0}+j B_{i k} \sin \Theta_{e, i k}^{0}\right], \\
Q_{e, i}^{0} & =\sum_{k \mid i k \in \mathcal{E}} V_{i}^{0} V_{k}^{0}\left[G_{i k} \sin \Theta_{e, i k}^{0}-j B_{i k} \cos \Theta_{e, i k}^{0}\right] .
\end{aligned}
$$

The subscript $(\cdot)_{e}$ denotes electrical quantity. The connection of a mechanical generator to the electric network is commonly modeled using the constant voltage behind transient reactance model, see [8]. Then, the real electric power output of machine $i$ is

$$
\begin{aligned}
& P_{e, i}=\left(V_{i}^{0}\right)^{2} Y_{i i} \cos \gamma_{i}+\sum_{k \mid i k \in \mathcal{E}} V_{i}^{0} V_{k}^{0} Y_{i k} \sin \left(\Theta_{e, i k}+\varphi_{i k}\right) \\
& =\left(V_{i}^{0}\right)^{2} G_{i}+\sum_{k \mid i k \in \mathcal{E}} V_{i}^{0} V_{k}^{0}\left[G_{i k} \cos \Theta_{e, i k}+j B_{i k} \sin \Theta_{e, i k}\right] \\
& :=P_{e, i i}+P_{e, i k}
\end{aligned}
$$

where $\varphi_{i k}:=\pi / 2-\gamma_{i k}$.

The local generator dynamics result from the mechanical swing equation

$$
M_{i} \ddot{\Theta}_{m, i}+D_{i} \dot{\Theta}_{m, i}=P_{m, i}-P_{e, i}(t):=P_{a, i}(t),
$$

where $\Theta_{m, i}$ is the mechanical rotor angle, $P_{m, i}$ the mechanical power input, which is assumed to be constant, $D_{i}$ is a damping constant, $P_{a, i}$ the accelerating power, $J_{i}$ the moment of inertia, $M_{i}:=J_{i} \omega_{0}$ the inertia constant, and $\omega_{0}$ the angular velocity of the rotating reference frame. Assuming the rotor angle being equivalent to the voltage angle behind transient reactance results in the relation $\Theta_{m, i} \triangleq \Theta_{e, i}:=\Theta_{i}$, see [8].

Remark 1: Referring to [1 (1a) (4), and (1b) (2), where active and reactive power are linked via voltages, and $\boldsymbol{x} \triangleq \Theta, \boldsymbol{y} \triangleq \boldsymbol{V}$.
The classical model for dynamics of a generator $i$ relative to an infinite bus, indexed as $(\cdot)_{\infty}$, is obtained by multiplying the swing equation (4) with $2 \dot{\Theta}_{i}$, assuming damping to be negligible $D_{i}=0$, see [8], and rewriting as

$$
\begin{aligned}
\frac{d}{d t}\left[\dot{\Theta}_{i}^{2}\right] & =\frac{2}{M_{i}} P_{a, i \infty} \dot{\Theta}_{i}, \\
\Rightarrow \dot{\Theta}_{i} & =\sqrt{\frac{2}{M_{i}} \int_{\Theta_{i}^{0}}^{\Theta_{i}} P_{a, i \infty} d \Theta .}
\end{aligned}
$$

Classically the infinite bus is a static approximation of a large utility or network that rests at constant voltage and frequency after any disturbance occurs. In (5), the integration limits depend only on $\Theta_{i}$, as $\Theta_{\infty}$ is a static quantity and $\Theta_{i}$ evolves relativ to it. Stationarity requires the local angular velocity $\dot{\Theta}_{i}$ in (5) either be zero (when acceleration due to $P_{a, i \infty}$ is zero) or opposing the rotor motion $\left(\dot{\Theta}_{i}(t)\right.$ is decreasing along $\Theta_{i}(t)$, and the rotor decelerates), so that $P_{a, i \infty} \leq 0$ determines convergent motion. Moreover, power balance for only one generator connected to an infinite bus assumes $\exists \Theta_{i}^{\max }: \int_{\Theta_{i}^{0}}^{\Theta_{\text {max }}} P_{a, i \infty} d \Theta=0$. This condition is known as equal area criterion [8]. Relaxing (5) such as to represent the relative dynamics against the aggregate nonstatic electric power system instead of an infinite bus, power balance does not only depend on local angles at generator $i$, as the reference angles at $k \neq i, \Theta_{k}$, can no more be considered as static. By that, a variable $\Theta_{i}^{\max }$ can no more be defined locally, but only in a global sense as it depends on all buses in the interconnected electric power system. Additionally, power is transported between buses $i$ and $k$ over branches $i k$. Capacity, and impeding characteristics of branches, and thus their contribution to global dynamics, depend continuously on the line length, i.e. the distance between $i$ and $k$. Due to this dependence of power flow and interaction on continuous distance in space we introduce a continuous spatial domain $\Omega \subset \mathbb{R}^{2}$ equipped with continuous spatial coordinates $z$. Generators are placed at $z(i)$, and local angles find their continuation over the branches $i k$ along curves in space $z_{i k}$, i.e. $\forall i, k \in \mathcal{V}, k \mid i k \in \mathcal{E}$

$$
\begin{aligned}
& \exists \tilde{\Theta}\left(z \in z_{i k}, t\right): \Theta_{i}(z(i), t)<\tilde{\Theta}<\Theta_{k}(z(k), t), \\
& \tilde{\Theta}(z(i), \cdot)=\Theta_{i}(\cdot), \text { and } \int_{z_{i k}} \frac{\partial \tilde{\Theta}}{\partial z} d z=\gamma_{i k}, \\
& \lim _{z \rightarrow z(k)} P_{e, i k}(\Theta(z(k)), \tilde{\Theta}(z))(t)=0 .
\end{aligned}
$$

From (6a) and (6b) $\tilde{\Theta}$ can be seen as the continuous interpolate between $\Theta_{i}$ and $\Theta_{k}$ over the branch along $z_{i k}$. Together with (6c) continuation of power flow means vanishing power exchange with vanishing distance, as angle differences, and by that potential differences vanish. Hence, $\tilde{\tilde{\Theta}}$ is the transport of the continuous angle distribution $\tilde{\Theta}(z, \cdot)$ over $\Omega$ in time, and $\Theta$ is its lumped parameter approximate. Referring to (5), we formally state the spatially enhanced version

$$
\dot{\Theta}_{i}=\sqrt{2 \int_{\Omega} \frac{P_{a, i}(\tilde{\Theta}(z))}{M_{i}(z(i))} d z .}
$$


describing the relative generator dynamics against all other continuously connected, spatially separated generators.

\section{B. Diffusive Coupling and Kuramoto Approximation}

Swing dynamics (4) have been shown to be related to diffusively coupled oscillator networks described by a Kuramoto model [5]. The classical Kuramoto model is the canonical form for synchronization schemes of phasecoupled oscillators. It is an all-to-all coupled, i.e. global information algorithm [9], where local information is exchanged between every subsystem at each instant in time

$$
\begin{aligned}
& \dot{\Theta}_{i}=\omega_{i}+\frac{1}{N} \sum_{k \in \mathcal{V}} K_{i k} \sin \Theta_{k i}=\omega_{i}+\frac{K}{N} \sum_{k \in \mathcal{V}} \sin \Theta_{k i} \\
& =\omega_{i}+K \Pi \sin \left(\Psi-\Theta_{i}\right), \bar{\Pi}=\Pi e^{j \Psi}=\frac{1}{N} \sum_{k \in \mathcal{V}} e^{j \Theta_{k}}
\end{aligned}
$$

The parameter $\omega_{i}$ is called natural frequency, the parameter $K_{i k}=K \in \mathbb{R}$ is a constant coupling gain, the constant $N$ denotes the cardinality of $\mathcal{V}, \sum_{k \in \mathcal{V}} \sin (\cdot)$ is the phase interaction function, the parameter $\Psi$ the mean phase, and the parameter $\Pi \in[0,1]$ is a measure of synchrony, i.e. phase uniformity gives $\Pi=1$ and maximum phase divergence yields $\Pi=0$, see [9], [10]. The pair $(\Psi, \Pi)$ is called mean field, and $8 \mathrm{~b}$ is obtained from multiplying $\bar{\Pi}$ with $e^{-j \Theta_{i}}$ and substituting the imaginary parts into (8a) [10]. Kuramoto dynamics are a special instance of diffusively coupled dynamics

$$
\begin{aligned}
& \dot{\Theta}_{i}=\omega_{i}+\Gamma_{i}(\mathcal{G}, K, \Theta)=\omega_{i}+\sum_{k \mid i k \in \mathcal{E}} \Gamma_{i k}\left(K_{i k}, \Theta_{i k}\right), \\
& \Gamma_{i k}\left(K_{i k}, \Theta_{i k}\right)=\frac{1}{2 \pi} \int_{0}^{2 \pi} \delta_{i}(\Psi) p_{i k}(\Psi, \Psi+\varpi) d \varpi
\end{aligned}
$$

Here, interaction is formally represented by the function $\Gamma_{i}$, and pairwise phase interaction defined by a function $\Gamma_{i k}$ relates to averaging of mutual phase perturbations defined by the perturbation function $\delta_{i}$ with a phase response curve $p_{i k}$ over a full phase cycle. Diffusive interaction as an averaging approach is valid when mutual perturbations stemming from interactions are small w.r.t natural frequencies, i.e. interaction is weak [10].

Remark 2: In (8) pairwise interaction over a graph is equivalent to local interaction with the mean field, as

$$
\Gamma_{i}(\mathcal{G}, K, \boldsymbol{\Theta}) \triangleq \sum_{k \mid i k \in \mathcal{E}} \Gamma_{i k}\left(K_{i k}, \Theta_{i k}\right) \triangleq \Gamma_{i}\left((\Psi, \Pi), K_{i}, \Theta_{i}\right)
$$

where $K_{i}:=\sum_{k \mid i k \in \mathcal{E}} K_{i k}$.

The modified version of $8 \mathrm{a}$ termed non-uniform $\mathrm{Ku}$ ramoto model has been applied to the synchronization problem in electric power systems in [5] as

$$
\dot{\Theta}_{i}=\frac{\omega_{i}}{D_{i}}-\sum_{k \in \mathcal{V}} \frac{P_{i k}^{0}}{D_{i}} \sin \left(\Theta_{i k}+\varphi_{i k}\right), \quad P_{i k}^{0}=P_{k i}^{0}
$$

The natural frequency $\omega_{i}:=P_{m, i}-V_{i}^{2} G_{i i}$ is associated to the $i$-th generator's effective power input in (4), and the symmetric coupling weights $P_{i k}^{0}:=V_{i}^{0} V_{k}^{0} Y_{i k}>0$ correspond to the maximum power transferred between two generators $i$ and $k$, where voltages relate to a static solution of (2). The original sparsity structure of the connected electric network is reduced to an effective all-to-all coupled graph with constant weights $K_{i k}=P_{i k}^{0} / D_{i}$. The quantities $P_{e, i i}, \gamma_{i i}$ are set to zero by convention [5]. It is termed non-uniform as the effective coupling gains and sinusoidal coupling functions are non-symmetric, i.e.

$$
\begin{aligned}
\frac{P_{i k}^{0}}{D_{i}} & \neq \frac{P_{i k}^{0}}{D_{k}}, \\
\sin \left(\Theta_{i k}+\varphi_{i k}\right)+\sin \left(\Theta_{k i}+\varphi_{k i}\right) & \neq 0 .
\end{aligned}
$$

In this paper we seek to find a Kuramoto-type approximation of (7) and interpret electric power grid dynamics on that basis.

\section{Spatially Embedded Kuramoto Dynamics}

We consider a symmetric, two-dimensional continuous domain $\Omega=\left\{\left(z_{1}, z_{2}\right) \mid z_{1}^{2}, z_{2}^{2}<R^{2}, R \in \mathbb{R}_{0}^{+}\right\}$. For the sake of simplicity, we assume here that $\sqrt{N} \times \sqrt{N}, \sqrt{N} \in \mathbb{N}$ generators are ordered in a square lattice graph $\mathcal{G}(\{1, \ldots, \sqrt{N}\} \times$ $\{1, \ldots, \sqrt{N}\},\{\{i k, \ln \}:|i-l|+|k-n|=1\})$, where $z(i) \triangleq i$ are the positions of generators that coincide with nodes of $\mathcal{G}$. Generators interact with each other through the network, i.e. local differences at $i$ first affect the continuous transmission system before a generator $k$ reacts.

\section{A. Properties of Interaction between Spatially Distributed Generators}

Due to propagation phenomena local dynamics interact with respect to distance-dependent, constant delays $\tau_{i k} \propto$ $d(i, k):=d(z(i), z(k)):=\left\|z_{i k}\right\|$. The regular lattice is spatially invariant, see [11], as translation results in the symmetry property

$$
\tau_{i(i+k)}=\tau_{i(i-k)} .
$$

A transmission delay induces a phase shift

$$
\left|\varphi_{i k}\right| \propto\left|\Theta_{i k}(t)-\left[\Theta_{i}\left(t-\tau_{i k}\right)-\Theta_{k}(t)\right]\right| \propto d(i, k) .
$$

The translational symmetry property of delayed interaction is related to rotation symmetry of phase-shifts as

$$
\varphi_{i(i+k)}=-\varphi_{i(i-k)} .
$$

Under the convention $P_{i i}, \gamma_{i i}=0$, see II-B (3) can be written as

$$
P_{e, i}=\sum_{k \in \mathcal{V}, k \neq i} P_{i k}^{0} \sin \left(\Theta_{i k}+\varphi_{i k}\right) \Rightarrow P_{i k}^{0}=\frac{P_{e, i k}}{\sin \left(\Theta_{i k}+\varphi_{i k}\right)},
$$

and hence, the coupling gain $K_{i k}=\frac{P_{i k}^{0}}{D_{i}}$ in (10) is related to power flow (2a) across branches as

$$
K_{i k}=\frac{P_{e, i k}^{0}}{D_{i} \sin \left(\Theta_{i k}^{0}+\varphi_{i k}\right)}=\text { const. }
$$

Here, we consider power flow being continuous in space, due to the continuation property of local angles over lines, 
see (6). By means of losses stemming from transfer conductances, $K_{i k}$ as coupling by power transfer between generators $i$ and $k$ becomes a distance-dependent continuous function that decreases with spatial distance. A spatial kernel function describing a local generator $i$ 's coupling with the spatially distributed generators $k \neq i$ over the electric interconnection structure is a two-dimensional Gaussian

$$
\mathrm{K}_{i}(z)=A e^{-\frac{z_{1}^{2}+z_{2}^{2}}{B}}>0,
$$

parameterized by constants $A$ and $B$, and centered at $z(i)$. Associating coupling kernels (17) to each generator $i$, centered at $z(i)$, the coupling $\mathrm{K}$ is no more constant in an absolute, i.e. an Eulerian reference frame, but constant from a local, i.e. Lagrangian view, as maximum coupling presides between least distant generators from the perspective of each moving generator $i$. An absolute coupling function can formally be stated in terms of a mean field description as in (8b), together with the interaction equivalences in Remark 2

$$
\Gamma_{i}\left(\mathrm{~K}(\Psi, \Pi)(z), \Theta_{i}\right) \triangleq \sum_{k \in \mathcal{V}} \Gamma_{i k}\left(\mathrm{~K}_{i}(z), \boldsymbol{\Theta}\right)
$$

where the distributed global mean phase $\Psi(z)$ evolves in space and time.

Other than in $\amalg$-B continuity and differentiability properties of the relative coupling kernel (17) allow to consider higher order effects, bearing for instance the natural interpretation of accompanying diffusion of power through the interconnection structure. In that respect, the Laplacian of the relative spatial coupling kernel is of interest

$$
\Delta \mathrm{K}_{i}(z):=\mathcal{L}\left(\mathrm{K}_{i}\right), \quad \mathcal{L}:=\frac{\partial^{2}}{\partial z_{1}^{2}}+\frac{\partial^{2}}{\partial z_{2}^{2}} .
$$

Remark 3: In view of (13) and (2) coupling (17) with $\mathrm{K}_{i}(d(i, k)) \sim \mathrm{K}_{i}\left(P_{i k}^{0}\right) \triangleq \mathrm{K}_{i}\left(\varphi_{i k}, V_{i}^{0}, V_{k}^{0}\right) \triangleq$ $\mathrm{K}_{i}\left(\Theta_{i k}, \tau_{i k}, V_{i}^{0}, V_{k}^{0}\right)$ respects both distance dependent losses and phase-shifts due to delays.

\section{B. Dynamics and Stationary Solutions}

Starting from (9) and using (17) with (18), dynamics under diffusive coupling can be written as

$$
\begin{aligned}
\dot{\Theta}_{i} & =\omega_{i}+\Gamma_{i}, \\
\Gamma_{i} & =-\left.\mathrm{K}(\Psi, \Pi)\right|_{z(i)} p_{i} \\
& \triangleq-\sum_{k \mid i k \in \mathcal{E}} \mathrm{K}_{i}(z) \circ p_{i k} \\
& =-\int_{\Omega} \mathrm{K}_{i}\left(z_{1}, z_{2}\right) p_{i}\left(\Theta_{i}, \varpi\left(z_{1}, z_{2}\right)\right) d\left(z_{1}, z_{2}\right) \\
& \propto-\int_{0}^{2 \pi} \mathrm{K}_{i}(z) p_{i}\left(\Theta_{i}, \varpi(z)\right) \frac{d z}{d \varpi} d \varpi .
\end{aligned}
$$

While the local to global interaction is formally stated in (20b) as interaction at $z(i)$ with some locally evaluated global mean field, in the step (20c) the more practical interaction over the graph is used. However, it is unclear how the distributional local coupling functions are to be connected with pair-wise phase interaction function over the graph. In the step towards 20d we took advantage of the homogeneous space and replace the sum over the symmetric lattic graph with a convolution integral. Here, the kernel function satisfies $\int_{\Omega} \mathrm{K}\left(z_{1}, z_{2}\right) d\left(z_{1}, z_{2}\right)=$ const., and $0 \geq$ $\mathrm{K}\left(z_{1}, z_{2}\right)$ and hence it is a stochastic kernel, operating on the continuous spatial domain. Finally, in 20e the domain of integration is mapped to the circle, due to radial symmetry in the system (point symmetry of the circle, and angular coordinates). Due to homogeneity, the Jacobian of the spatial parameterization $d z / d \tilde{\tau}=$ const everywhere, and without loss of generality it is set to one.

Remark 4: Interestingly the family of closed curves $s:=$ $e^{j t}+$ const $t \in[0,2 \pi) \triangleq \partial \Omega(R=1)$, after a conformal mapping to the unit disk. By that, (20d $\sim 2$ assumes the integrand to satisfy a mean-value property, being related to the calculus of variations and the divergence theorem, see for instance [12]. The divergence theorem relates to the existence of a conservation law, that acts as generator of a vector field that induces conservative flow [13]. Such families of trajectories are minimzers of an availabe energy functional. Assuming sinusoidal phase response as in (8a), and applying the trigonometric identity $\sin (a \pm b)=\sin (a) \cos (b) \pm$ $\cos (a) \sin (b)$ yields

$$
\begin{aligned}
\Gamma_{i}= & -\int_{\Omega} \cos \Theta_{i} \mathrm{~K}_{i}(z) \sin \left(\Theta_{i}-\varpi(z)\right) d z \\
& +\int_{\Omega} \sin \Theta_{i} \mathrm{~K}_{i}(z) \cos \left(\Theta_{i}-\varpi(z)\right) d z \\
= & -\cos \Theta_{i} \int_{0}^{2 \pi} \mathrm{K}_{i}(z) \sin \left(\Theta_{i}-\varpi\right) d \varpi \\
& +\sin \Theta_{i} \int_{0}^{2 \pi} \mathrm{K}_{i}(z) \cos \left(\Theta_{i}-\varpi\right) d \varpi \\
:= & \Gamma_{i, a t t}(\mathrm{~K}, \Theta)+\Gamma_{i, r e p}(\mathrm{~K}, \Theta) .
\end{aligned}
$$

Here, $\Gamma_{i, a t t}$ refers to attractive (synchronizing) interaction, and $\Gamma_{i, r e p}$ refers to repulsive (desynchronizing) interaction, see [5], [8], and 21b is the spatial interaction function for the Kuramoto dynamics 20a studied in IV The Kuramoto approximation (10) is a special case of the spatio-temporal Kuramoto dynamics (21). Expanding the interaction in (10) results in

$$
\dot{\Theta}_{i}=\frac{\omega_{i}}{D_{i}}-\sum_{k \in \mathcal{V}} K_{i k}\left[\cos \varphi_{i k} \sin \Theta_{i k}+\sin \varphi_{i k} \cos \Theta_{i k}\right]
$$

which is (21) after evaluation only at buses. Hence, (22) is a lumped parameter version of dynamics with distributional interaction function in (21).

Remark 5: In 22 interaction precisely separates into pairwise coupling gains, phase shifts, and the harmonic terms which are multiplied appropriately. In contrast to this vector case, the continuous approach (21) intertwines coupling gains and delayed phase interaction in the context of convolution integrals. A vector approximates a continuous function. Any error resulting from such a lumpedparameter approximation will affect the global dynamics in the convolutive setting due to conservation properties of the interaction with the stochastic kernel over $\Omega$. Here, errors 
will be induced on a numerical basis reflecting discrepancies of the (locally) finite-state but infinite domain setting.

A stationary solution $\Theta^{s}(t)$ is defined in terms of the set of equivalent equilibria

$E_{\Theta^{s}}:=\left\{\boldsymbol{\Theta} \in \mathbb{T}^{N} \mid \boldsymbol{\Theta}=\boldsymbol{\Theta}^{s}+\lambda \mathbf{1}, \lambda \in \mathbb{R}\right\}, \quad \mathbb{T}^{N}:=\mathbb{R}^{N} / \mathbb{Z}^{N}$,

where $\boldsymbol{\Theta}^{s}$ satisfies

$$
\omega_{i}=-\sum_{k \in \mathcal{V}} \Gamma_{i k}\left(\mathrm{~K}_{i}(z), \Theta^{s}\right)
$$

Assuming identical generators $\omega_{i}=\omega_{k}:=\hat{\omega}=$ const, condition (24) corresponds to vanishing interaction along trajectories $\boldsymbol{\Theta}(t)=\hat{\omega} t \mathbf{1}+\boldsymbol{\Theta}^{s}$. In that moving frame we define the non-dimensional global mean phase frustration as

$$
\chi_{\Omega}(\tilde{\Theta}(t)):=\frac{\hat{\chi}_{\Omega}(\tilde{\Theta})}{N \hat{\chi}_{\Omega}(\tilde{\Theta})}, \quad \hat{\chi}_{\Omega}:=\sum_{i \in \mathcal{V}}\left|\hat{\Gamma}_{i, a t t}+\hat{\Gamma}_{i, r e p}\right| .
$$

At $\Pi=1, \chi_{\Omega} \approx 0$, however $\chi_{\Omega}$ does not relate to a degree of synchrony, but it is a measure for aggregated local coupling tension, where local tension manifests in local phase angle velocity. Stationarity (24) and independence in the cyclic coordinate $\hat{\omega}$ is equivalent to the conditions

$$
\begin{aligned}
& \left\|\chi_{\Omega}(t)\right\|=0, \quad \text { s.t. } \frac{\partial \chi_{\Omega}}{\partial \hat{\omega}}=0 \Leftrightarrow \chi_{\Omega}(t)=\text { const }, \\
& \left(\hat{\Gamma}_{i, a t t}+\hat{\Gamma}_{i, r e p}\right)=0 \Leftrightarrow\left|\Gamma_{i, a t t}+\Gamma_{i, r e p}\right|=\text { const. }
\end{aligned}
$$

Due to the distributional setting the inner product and thus the norm is evaluated via a spatial integral. Thus the norm vanishes over that integral, but may locally be non-zero, as long as all non-zero contributions constitute some harmonic function over the integral domain. Condition (26b) suggests quasi-stationarity as varying $\chi_{\Omega}(t)$ where in intervals $n \frac{2 \pi}{\hat{\omega}} \leq$ $t<(n+1) \frac{2 \pi}{\hat{\omega}}, n \in \mathbb{N}$ attraction and repulsion balance up to a constant to yield arbitrary small oscillations as follows: Frequency entrainment corresponds to exponential synchronization to some bounded frequency $\dot{\Theta}^{s} \in\left[\dot{\Theta}^{\min }, \dot{\Theta}^{\max }\right]$, and phase locking to positive invariance of the set of bounded phase differences $\left\{\Theta \in \mathbb{T}^{N}: \max _{i k \in \mathcal{E}}\left|\Theta_{i k}\right| \leq \gamma\right\}$, see [5]. Assume exponentially small differences of the form $\Theta_{i}-\Theta_{i-k}=\Theta_{i}-\Theta_{i+k}$, and substituting into the lumped parameter dynamics (22) gives together with (14) for the sinusoidal phase interaction

$$
\begin{aligned}
& \sum_{k=1}^{N} \cos \varphi_{i k} \sin \left(\Theta_{i}-\Theta_{k}\right) \\
& =\sum_{k=1}^{N / 2} \cos \varphi_{i(i-k)} \sin \Theta_{i(i-k)}+\sum_{k=1}^{N / 2} \cos \varphi_{i(i+k)} \sin \Theta_{i(i+k)} \\
& =\sum_{k=1}^{N / 2} \cos \varphi_{i(i+k)} \sin \Theta_{i(i+k)}+\sum_{k=1}^{N / 2} \cos \varphi_{i(i+k)} \sin \Theta_{i(i+k)} \\
& =\sum_{k=1}^{N} \cos \varphi_{i k} \sin \left(\Theta_{i}-\Theta_{k}\right),
\end{aligned}
$$
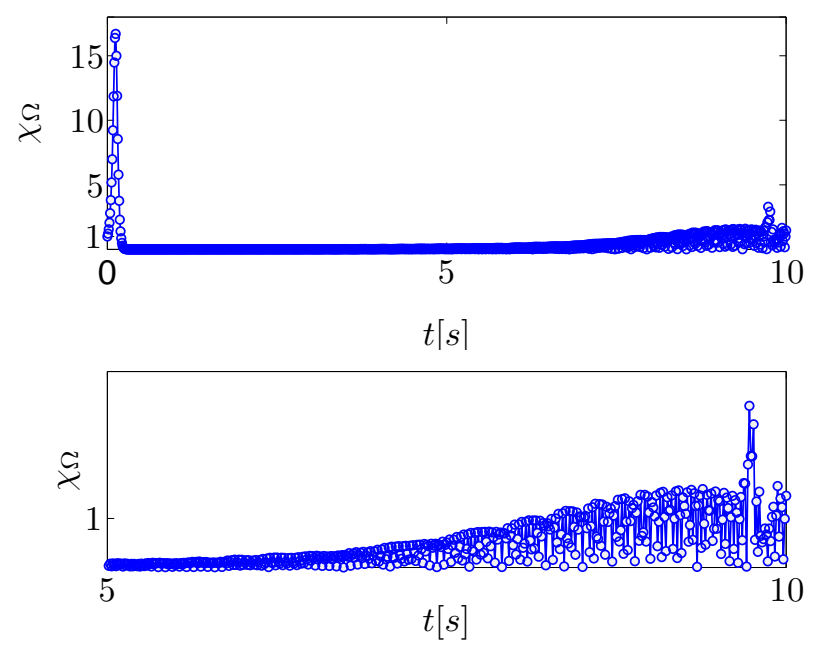

Fig. 1. Global phase frustration over time; $R=7.5, d t=0.01 \mathrm{~s}$

while the cosine interaction term expanded similarly results in

$$
\sum_{k=1}^{N} \sin \varphi_{i k} \cos \left(\Theta_{i}-\Theta_{k}\right)=0 .
$$

A stationary solution of (22), and (21) then has the form

$$
\dot{\Theta}_{i}=\omega+\sum_{k \in \mathcal{V}} K_{i k} \cos \left(\varphi_{i k}\right) \sin \left(\Theta_{i}-\Theta_{k}\right) .
$$

This corresponds to a non-trivial traveling wave solution, which is stabilized in a balance between attraction due to $K_{i k} \sin \left(\Theta_{i}-\Theta_{k}\right)$ terms and impeding terms $K_{i k} \cos \left(\varphi_{i k}\right)$, where pairwise phase-interaction contributes homogeneously to a global rotatory motion (27). This type of stationarity is called quasi-stationarity.

Remark 6: Note that when the limit $N \rightarrow \infty$ is taken, then the continuous domain will be densly filled with oscillators, so that local phase difference will vanish, too, i.e. $\mid \Theta_{i k} \rightarrow 0$ as $\tau_{i k} \rightarrow 0$. When $N$ rests finite small mismatches due to nonvanishing delays will in general always be present.

\section{NUMERICAL StUdy}

In the following, $20 \times 20$ Kuramoto oscillators are simulated, being ordered in a regular lattice, where each square relates to one generator phase. Dynamics evolve under interation $21 \mathrm{~b}$, and $d t$ refers to the discrete time step size. The system is initialized with uniform natural frequencies $\omega_{i}=2 \pi / s$ and the phase angles are initialized uniform random. We apply the parameterization $A=R / \sqrt{N}$, and $B=R^{2}$.

In Fig 1 a typical transient behavior of 21) is depicted. The initial phase frustration of value 1 is first strongly amplified until fast convergence sets in. The converged solution is prone to strong fluctuations in global phase frustration, which eventually leads to sharp peaks as can be seen at $9.8 s$.

In Fig 2 a snapshot at that critical moment is depicted. Although the global phase frustration exceeds the initial value of 1 , phase angle difference is globally bounded and 
small. Global stress manifests in periodic, increased local coupling tension. These fluctuations increase in frequency
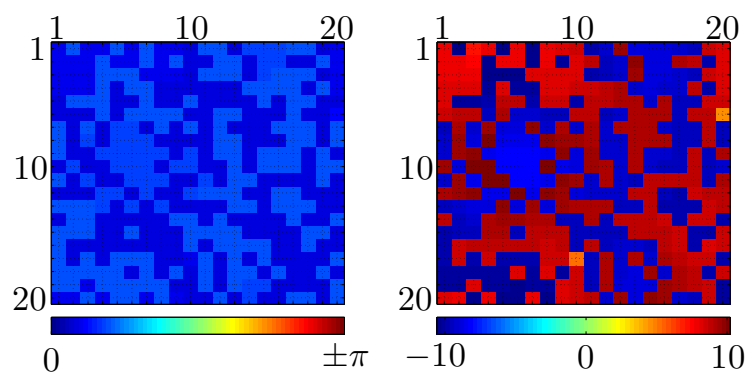

Fig. 2. Phase angles (left), and local coupling tension (right) in $[\mathrm{rad} / \mathrm{s}]$, corresponding to Fig 1 at $t=9.8 \mathrm{~s} ; d t=0.01 \mathrm{~s}$

with refined step width $d t$, but do not vanish and do not change in magnitude. According to (15), fluctuations in the interaction function are related to power flow variations. In fact, power flow oscillations are constantly observed in steady state operation of large power systems, see [8]. In the same sense as synchronization dynamics are studied on grounds of a static power balance solution, next we illustrate the tendency of angle dynamics due to power diffusion at $t_{0}$. For that we apply an interaction function proportional to the Laplacian (19) of the original coupling kernel. In Fig 3 a stationary solution is depicted. Traveling waves move across the spatial domain, with the directions indicated in the left illustration. Comparing with the coupling tension on the right, it can be seen, that locally the fronts are moving according to a spatial gradient in adjacent velocity differences. Hence, the global traveling wave solution organizes not from allto-all interaction but from generator dynamics being driven by only local coupling tension in stationary state. From an energetic point of view and related to the physics of electric power grids, strong coupling tension as in Fig 1 should be minimized simultaneously by damping due to inertias and global oscillations corresponding to power diffusion. An
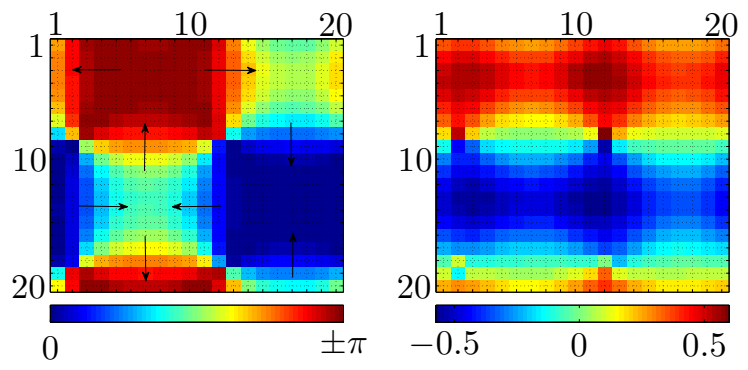

Fig. 3. Phase angles (left), and local coupling tension (right) in $[\mathrm{rad} / \mathrm{s}]$ due to interaction with $\mathcal{L}\left(\mathrm{K}_{i}\right)$

observation in that direction is that the transient overshoot in global tension initially is much lower for the case pictured in Fig 3, as shown in Fig 4 In control, transient overshoot corresponds to good tracking behavior, i.e. reactivity, but at the same time it causes sensitivity to disturbances. Hence a robust stationary solution, i.e. phase angles and global phase frustration are both bounded simultaneously, consists in a trade-off of the behavior due to interaction with $\mathrm{K}_{i}$ and $\mathcal{L}\left(\mathrm{K}_{i}\right)$. By that, peaks in Fig 1 above a certain limit should physically manifest in spatial drift as in Fig 3 , leading to power flow variations that interfere with constant base power flow. Small non-vanishing fluctuations are also observed for

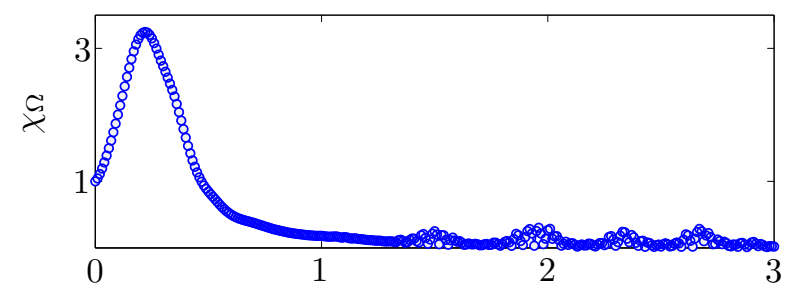

Fig. 4. Global phase frustration over time corresponding to Fig 3 at $t=$ $2.4 s ; R=7.5, d t=0.01 s$

interaction with $\mathcal{L}\left(\mathrm{K}_{i}\right)$, as shown in Fig 4 , however, these are less aggressive with respect to sudden peaking phenomena. Interestingly, the traveling wave solution minimizes phase frustration to the same level as a quasi-homogeneous solution as in Fig 112 Note the smallness of local coupling tension in Fig 3 relative to those in Fig 2 and compare with the magnitudes of the respective transient overshoots. Transient overshoots result from instantaneous coupling strength as can be seen from Fig 5 An increase in $R$ yields an increase of

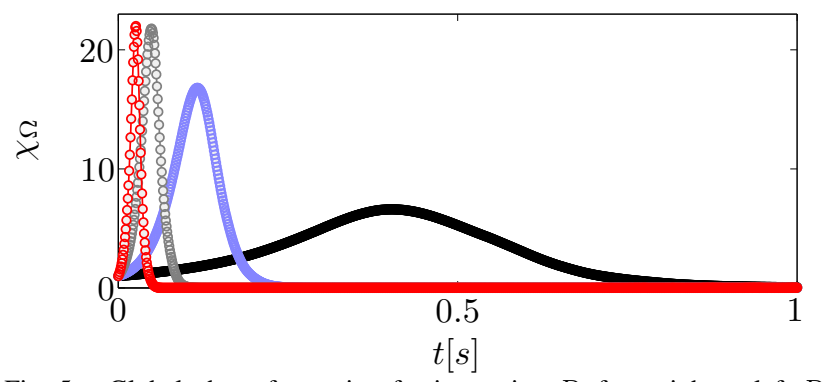

Fig. 5. Global phase frustration for increasing $R$; from right to left $R=$ $5,7.5,10,12.5 ; d t=0.001 s$

the amplification factor $A$. At the same time the width of the Gaussian coupling function $\mathrm{K}_{i}$ increases as the number of generators $N$ stays constant. Consequently the degrading of coupling strength with distance is lowered. The peak magnitude appears to be bounded despite further increase in $R$.

Remark 7: Non-uniform Kuramoto dynamics (10) are related to those of network models for power systems via a toplogical equivlance only of the equilibria, not the full dynamics [14]. In that light the phenomenon of peaking becomes more interesting, because it develops on grounds of an "almost" (i.e. up to numerical errors) phase synchronized (equilibrium) solution. In the same context, strong transient overshoot can be interpreted as sensitivity of an equilibrium subjected to disturbances represented in form of the chosen initial condition. 


\section{Conclusion and Future Outlook}

Motivated by local generator dynamics relative to an infinite bus, and recent advances in modeling electric power grid dynamics in a Kuramoto oscillator framework, we approximate electric power grid dynamics in terms of a spatially embedded Kuramoto model. Interaction of spatially separate generators is mediated by the global electric transmission system. In numerical simulations we observe predicted quasistationary behavior, and additionally spontaneous peaking phenomena.

Future work is related to analyzing dynamic stability in terms of transport of spatial energy distributions and their gradients.

\section{REFERENCES}

[1] E. Grebe, J. Kabouris, S. L. Barba, W. Sattinger, and W. Winter, "Low frequency oscillations in the interconnected system of continental europe," in Power and Energy Society General Meeting, 2010 IEEE, pp. $1-7,2010$.

[2] M. Parashar, J. Thorp, and C. Seyler, "Continuum modeling of electromechanical dynamics in large-scale power systems," IEEE Transactions on Circuits and Systems: Fundamental Theory and Applications, vol. 51, no. 9, pp. 1848-1858, 2004.

[3] J. Thorp, C. Seyler, and A. Phadke, "Electromechanical wave propagation in large electric power systems," IEEE Transactions on Circuits and Systems: Fundamental Theory and Applications, vol. 45, no. 6, pp. 614-622, 1998.

[4] A. Bergen and D. Hill, "A structure preserving model for power system stability analysis," IEEE Transactions on Power Apparatus and Systems, vol. PAS-100, pp. 25-35, 1981.

[5] F. Dörfler and F. Bullo, "Synchronization and transient stability in power networks and non-uniform kuramoto oscillators," in American Control Conference, 2010.

[6] M. D. Ilic, "From hierarchical to open access electric power systems," Proceedings of the IEEE - Vol. 95, No. 5, May 2007, vol. 95, no. 5, pp. 1060-1084, 2007.

[7] E. Allen and M. Ilic, "Interaction of transmission network and load phasor dynamics in electric power systems," IEEE Transactions on Circuits and Systems I: Fundamental Theory and Applications, vol. 47, pp. 1613-1620, 2000.

[8] P. Anderson and A. Fouad, Power System Control and Stability. John Wiley and Sons, 2003.

[9] L. Scardovi, A. Sarlette, and R. Sepulchre, "Synchronization and balancing on the n-torus," Systems \& Control Letters, vol. 56, no. 5, pp. 335-341, 2007.

[10] M. Breakspear, S. Heitmann, and A. Daffershofer, "Generative models of cortical oscillations: neurobiological implications of the kuramoto model," Frontiers in Human Neuroscience, vol. 4, pp. 1-14, 2010.

[11] B. Bamieh, F. Paganini, and M. Dahleh, "Distributed control of spatially invariant systems," IEEE Transactions on Automatic Control, vol. 47, pp. 1091-1107, 2002.

[12] J. Jacobsen, "As flat as possible," SIAM REVIEW, vol. 49, no. 3, pp. 491-507, 20007.

[13] V. Arnold, Mathematical Methods of Classical Mechanics. Springer, 1989.

[14] F. Dörfler and F. Bullo, "Topological equivalence of a structurepreserving power network model and a non-uniform kuramoto model of coupled oscillators," in IEEE Conference on Decision and Control, 2011. 